\title{
Electrical Transport Properties of Multilayered Single-Walled Carbon Nanotube Films
}

\author{
Yanli Zhao and Wenzhi Li \\ Department of Physics, Florida International University, Miami, FL 33199, USA \\ Correspondence should be addressed to Wenzhi Li, wenzhi.li@fiu.edu \\ Received 3 December 2011; Accepted 17 January 2012 \\ Academic Editor: Rajaram S. Mane
}

Copyright () 2012 Y. Zhao and W. Li. This is an open access article distributed under the Creative Commons Attribution License, which permits unrestricted use, distribution, and reproduction in any medium, provided the original work is properly cited.

An improved layer-by-layer vacuum filtration method was adopted for the fabrication of single-walled carbon nanotube (SWCNT) films aiming at a series of SWCNT films with controllable thickness and density. The electrical transport properties of the multilayered SWCNT films have been investigated. With the constant film density, the decrease of the layer number of the SWCNT film results in an increase of the temperature coefficient of resistance (TCR). SWCNT film with 95\% metallic nanotubes has shown a lower TCR than that of the SWCNT films with a low percentage of metallic nanotubes. The effect of thermal annealing and subsequent acid $\left(\mathrm{HNO}_{3}\right)$ treatment on the electrical properties of the SWCNT films has also been investigated.

\section{Introduction}

Even though single-tube devices based on single-walled carbon nanotubes (SWCNTs) have excellent properties [1-3], these devices are usually fabricated randomly since it is very difficult to find two identical SWCNTs through currently available technology. Currently, the available technologies for SWCNT synthesis usually produce a mixture of nanotubes with varying diameters [4-8]. An as-prepared SWCNT can be either metallic or semiconducting depending on its chirality. In general, one-third of the synthesized SWCNTs are metallic, while the other two-thirds are semiconducting [9]. Even though the separation of metallic SWCNTs from a mixture of both metallic and semiconducting SWCNTs has recently become possible [10], the repeatable fabrication of two identical SWCNT devices is further than a near future work. From the application aspect, SWCNT films which contain SWCNTs with all kinds of chiralities are very attractive, since they suppress the differences among individual nanotubes.

Low temperature measurements may give some insight into the transport mechanism of SWCNT films, which could lead to an optimal SWCNT film with designed electrical properties [11]. In addition to being a potential replacement for ITO as a conductive and transparent electrode material, SWCNT thin films have also attracted a lot of attention recently due to their large bolometric photoresponse and high temperature coefficient of resistance (TCR) [12]. High TCR SWCNT films are highly desired for its potential application in infrared (IR) sensor. Vacuum filtration has been found as an effective approach for repeatable fabrication of single-walled carbon nanotube (SWCNT) films with a designed thickness and film density [1]. In this work, an improved layer-by-layer vacuum filtration method was adopted for the fabrication of SWCNT films aiming at a series of SWCNT films with controllable thickness and density. Decrease of the thickness of the SWCNT film with a constant film density will result in an increase of the TCR and a large resistance. The effect of thermal annealing and $\mathrm{HNO}_{3}$ treatment on the electrical properties of SWCNT film has also been discussed. Aiming to ultimately produce an optimal SWCNT film with a high TCR, our experiment provides insight into the design and selection of SWCNT films.

\section{Experiments}

In this research, we used commercially available HiPco purified SWCNTs and high purity (95\%) metallic SWCNTs from http://www.NanoIntegris.com. The HiPco SWCNTs are mixture of metallic and semiconducting nanotubes. Since no preferential growth for metallic and semiconducting SWCNTs is found in the HiPco process, it is reasonable to believe 
TABLE 1: The fabrication parameters, thickness, sheet resistance, and TCR at $300 \mathrm{~K}$ of the SWCNT thin films.

\begin{tabular}{|c|c|c|c|c|}
\hline Sample name & Sample Description & Thickness (nm) & $\begin{array}{c}\text { Sheet resistance at } \\
\text { room environment } \\
(\Omega / \square)\end{array}$ & TCR (at $300 \mathrm{~K}$ ) \\
\hline 1 & $1 \mathrm{~mL}$ SWCNT suspension, 2 layers & 45 & 45780 & -0.146 \\
\hline 2 & $2 \mathrm{~mL}$ SWCNT suspension, 1 layer & 35 & 10750 & -0.112 \\
\hline 3 & $2 \mathrm{~mL}$ metallic SWCNT suspension, 1 layer & 35 & 5693 & -0.060 \\
\hline 4 & $2 \mathrm{~mL}$ SWCNT suspension, 3 layers & 105 & 3210 & -0.088 \\
\hline $2 \mathrm{a}$ & Sample 2 after annealing at $300^{\circ} \mathrm{C}, 12$ hours & 35 & 104500 & -0.136 \\
\hline $4 \mathrm{a}$ & Sample 4 after annealing at $300^{\circ} \mathrm{C}, 12$ hours & 105 & 24100 & -0.101 \\
\hline $2 \mathrm{aN}$ & Sample 2a after $\mathrm{HNO}_{3}$ treatment & 35 & 19780 & -0.055 \\
\hline $4 \mathrm{aN}$ & Sample 4a after $\mathrm{HNO}_{3}$ treatment & 105 & 1012 & -0.0186 \\
\hline
\end{tabular}

that about one-third of the mixed HiPco SWCNTs could be metallic nanotubes [9]. The NanoIntegris SWCNTs has 95\% metallic SWCNTs which was extracted from mixed SWCNTs via an available process called density gradient centrifugation (DGC) as reported in [13]. When the samples were purchased, the NanoIntegris SWCNTs were already dispersed in aqueous solution with some water-soluble surfactants and the HiPco SWCNTs were in dry powder form. To make an aqueous solution of the HiPco SWCNTs, a surfactant (Triton-100) was used to help to disperse the nanotubes, followed by sonication process and a centrifugation process. The SWCNTs powder was dispersed in aqueous solutions of $1 \%$ v/v surfactant (Triton-100) via 1-hour ultrasonication and 0.5 -hour centrifugation. The concentration of the obtained HiPco suspension is about $5 \times 10^{-3} \mathrm{mg} / \mathrm{mL}$. The well-dispersed NanoIntegris SWCNT suspension was diluted to the same concentration as that of the obtained HiPco SWCNT suspension, further sonication and centrifugation process were not needed for the NanoIntegris SWCNT suspension. To prepare a nanotube film, an improved layer-by-layer vacuum filtration method $[14,15]$ was adopted to make singleand multilayered SWCNT films. The dispersed HiPco and NanoIntegris SWCNT suspensions were vacuum-filtered onto $0.1 \mu \mathrm{m}$ pore size mixed cellulose ester membrane (Millipore) and followed by washing with copious quantities of deionized water to remove the surfactants (Triton-100 and other surfactants if any). To transfer the nanotube film to a glass substrate, the still wet membrane was placed onto the substrate with film side down, which makes the nanotube film intimately contact with the glass substrate. The assembly was subsequently covered with a piece of clean filter paper to absorb the moisture and compressed with a $2 \mathrm{~kg}$ mass to keep the thin film flat for overnight. Then, the dried membrane was simply peeled off from the membrane/nanotube/glass sample. The density of each layer of the multilayered SWCNT films can be controlled by the amount of SWCNT in the suspension, and the multilayered SWCNT films have been prepared by a layer-by-layer vacuum filtration method. The so-called layer-by-layer method is described as follows: after the first layer of SWCNTs has been transferred to the glass substrate, a second vacuum-filtrated layer of SWCNTs will be transferred on the first layer, and the third vacuum-filtrated layer will be transferred on the second layer, and so on. In the multilayered SWCNT film, the SWCNTs in the same layer may be interwoven while the SWCNTs at the contact region of two adjacent layers will not interweave but a simple contact. In this experiment, to investigate the effect of the layer structure, density, and type of SWCNTs on the electrical properties of the SWCNT films, the following SWCNT film samples are prepared and tabulated in Table 1. Sample 1 has two layers, each layer was prepared using $1 \mathrm{~mL}$ HiPco SWCNT solution; sample 2 has only one layer prepared from $2 \mathrm{~mL}$ HiPco SWCNT solution; sample 3 has one layer prepared from $2 \mathrm{~mL}$ NanoIntegris metallic SWCNTs; sample 4 has three layers, each layer was prepared from $2 \mathrm{~mL}$ HiPco SWCNT solution. The as-prepared SWCNT films were thoroughly cleaned in acetone and then in ethanol for future electrical property measurements. Post treated samples (samples $2 \mathrm{a}-4 \mathrm{aN}$ ) were prepared by annealing the asprepared SWCNT films at $300^{\circ} \mathrm{C}$ in an Ar gas environment for 24 hours with and without subsequent $\mathrm{HNO}_{3}$ treatments. The $\mathrm{HNO}_{3}$ treatment was carried out by submerging the annealed nanotube/glass sample in $12 \mathrm{M} \mathrm{HNO}_{3}$ for $0.5 \mathrm{hrs}$.

Atomic force microscopy (AFM) topography images of the SWCNT thin films were acquired in the tapping mode using a Multimode AFM (Veeco Instruments). AFM was also used to measure the thickness of the SWCNT films. During the measurement, the AFM tip scans across the lateral edge between SWCNT film and the bare glass substrate. The scan range is large enough (more than $50 \mu \mathrm{m}$ ) to avoid the inaccuracy originating from the difference between the SWCNT film's edge and its inner part. The vertical distance (height) between the step of SWCNT film and the bare glass substrate was determined. Several points have been measured by scanning from SWCNT film to glass substrate to get an average height with a scan rate of $0.5 \mathrm{~Hz}$. The thickness of SWCNT films is defined as the average height obtained from the AFM measurements. The root-mean-square (RMS) roughness of the SWCNT film surface is typically less than $10 \mathrm{~nm}$.

The strip electrodes on the SWCNT thin film were painted using commercially available Ag paste (Ted Pella Inc.), then silver wire was used to contact the Ag strip electrodes and the sample stage of a cryostat (VPF-500, Janis Research) for electrical property tests. To measure the contact resistance, the same-sized strip electrodes with different spacing between the adjacent ones were designed on the same SWCNT film. In this work, the width of the SWCNT films 
was $5 \mathrm{~mm}$, and the spacing between the adjacent electrodes varied from $2 \mathrm{~mm}$ to $8 \mathrm{~mm}$. All electrical property tests were conducted using a Keithley 4200 SC semiconductor analyzer. The resistance between the electrodes with different spacing was then measured and used to determine the contact resistance of the silver/nanotube contact and the intrinsic resistance of the SWCNT films $[1,16]$. For comparison, the electrical properties of some SWCNT films have also been measured with four-point method. Our measurements showed that the contact resistance was about 10 times smaller than the intrinsic resistance of the SWCNT film.

\section{Results and Discussion}

Table 1 tabulated the detailed information of all SWCNT films used in this experiments. Sample 1 is a two-layered SWCNT film which was prepared with a layer-by-layer process as mentioned in the experimental part. During the process, $1 \mathrm{~mL}$ SWCNT suspension was used for each layer of sample 1. Sample 2 is a single-layer SWCNT film which was prepared with $2 \mathrm{~mL}$ SWCNT suspension. The thickness of the obtained films is $45 \mathrm{~nm}$ for sample 1 , and $35 \mathrm{~nm}$ for sample 2. Considering the same film dimension, the same total amount of SWCNTs used, and the different thickness for these two samples, it is easily to know that sample 1 has a lower density than that of sample 2. Comparison of sample 1 with sample 2 reveals that the decrease of the density of SWCNT film results in the increase of the sheet resistance in room environment from 10750 to $45780 \Omega / \square$, even though the same amount of SWCNT suspension was used during the vacuum filtration for the two samples. Sample 3 is a singlelayer SWCNT film prepared with $2 \mathrm{~mL}$ SWCNT suspension containing high metallic SWCNT content. If we ignore the diameter difference of metallic and semiconducting SWCNTs, it is reasonable to believe that samples 2 and 3 have the same thickness and density since we use the same volume of SWCNT suspension with the same concentration. In the error range of our experiment, it does show the same thickness of these two samples $(35 \mathrm{~nm})$ measured by AFM. The sheet resistance of sample 3 is nearly half of that of sample 2 due to the higher metallic SWCNT content (95\%) in sample 3 than that $(33.3 \%)$ in sample 2. Sample 4 is a 3layered sandwich SWCNT film, in which every layer is exactly the same as that in sample 2. The thickness of sample 4 is $105 \mathrm{~nm}$, which is 3 times as thick as that of sample 2. It is expected that the measured sheet resistance of sample 4 should be one-third of that of sample 2. However, we find that the resistance of sample 4 is slightly lower than the expected value, as shown in Table 1. It is well known that the conductivity of SWCNT film is determined by percolation theory [1]. A universal power law of the form $\sigma \propto\left(p-p_{c}\right)^{\alpha}$ is used to determine the relation between the conductivity $(\sigma)$ and the concentration of conducting channels $(p)$ near the percolation threshold $\left(p_{c}\right)$. The critical exponent $\alpha$, which provides an index of the system dimensionality, is 1.3 and 1.94 in theory for ideal 2D and 3D systems. In our experiment, the SWCNT film system dimensionality changes from

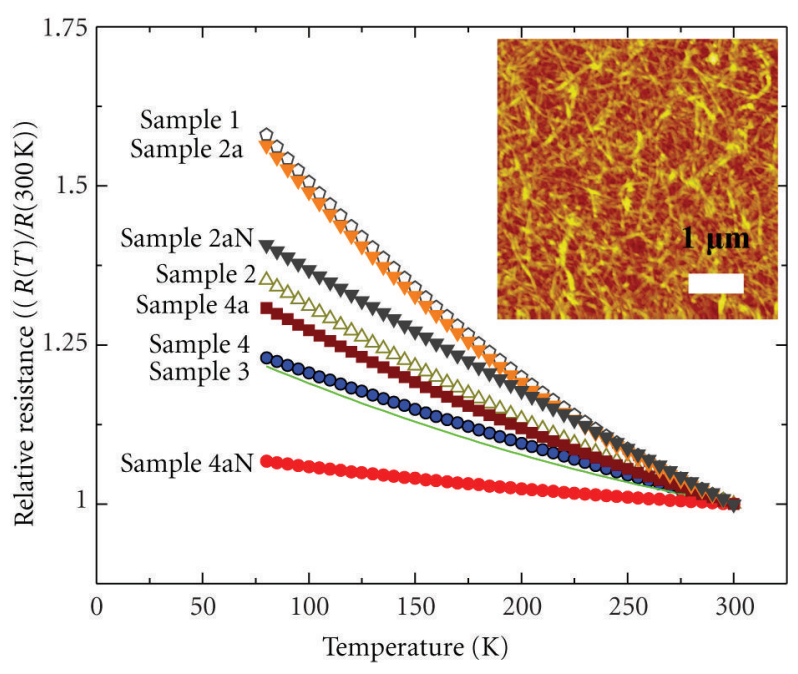

FIGURE 1: The change of relative resistance $(\mathrm{R}(\mathrm{T}) / \mathrm{R}(300 \mathrm{~K}))$ with temperature for samples $1-4 \mathrm{aN}$ at temperature of 78-300 K. Inset shows an AFM image of a typical SWCNT film.

$2 \mathrm{D}$ in sample 2 to $3 \mathrm{D}$ in sample 4 , which may account for the smaller resistance value for sample 4 than the expected value.

Sample $2 \mathrm{a}$ is obtained by annealing sample 2 at $300^{\circ} \mathrm{C}$ in an Ar gas environment for 24 hours, comparison of sample $2 a$ with sample 2 shows that the annealing treatment results in a rapid increase in the sheet resistance (from $10750 \Omega / \square$ to $104500 \Omega / \square$ ) at room temperature. The significant influence of annealing on the resistance of the SWCNT film was also observed in samples 4 and $4 \mathrm{a}$, as shown in Table 1. The experimental results indicate clearly that the annealing will result in the increase of the resistance of SWCNT films. On the contrary, further $\mathrm{HNO}_{3}$ treatment leads to the obvious decrease of sheet resistance. As shown in Table 1, the sheet resistance of sample 2a was $104500 \Omega / \square$, but after further acid treatment the resistance was decreased to $19780 \Omega / \square$ in sample $2 \mathrm{aN}$. Similarly, the further acid treatment of sample $4 \mathrm{a}$ also resulted in a huge decrease of resistance from $24100 \Omega / \square$ in sample $4 \mathrm{a}$ to $1012 \Omega / \square$ in sample $4 \mathrm{aN}$. The resistance decrease after acid treatment can be ascribed to the charge transfer [17-19] in the SWCNT films. The $\mathrm{HNO}_{3}$ oxidation will result in carboxyl $(-\mathrm{COOH})$ groups which will be attached to the defects sites and the SWCNT ends. The $\mathrm{COOH}$ groups are electron acceptors and they will accept electrons transferred from SWCNTs. As a result, the density of holes in the p-type SWCNTs will increase and the separation between the Fermi level and the valence band will also decrease. Therefore, the electrical resistance of the SWCNT films after $\mathrm{HNO}_{3}$ treatment will decrease [17-19].

Figure 1 shows the change of relative resistance $(\mathrm{R}(\mathrm{T}) /$ $\mathrm{R}(300 \mathrm{~K})$ ) with temperature for samples $1-4 \mathrm{aN}$ at the temperature range $80 \mathrm{~K}-300 \mathrm{~K}$. The inset in Figure 1 shows a typical AFM image of the SWCNT films. A nanoscale porous network was formed due to the tubular structure of the carbon nanotubes. The root mean square (rms) roughness for the SWCNT film calculated from AFM data is about $7 \mathrm{~nm}$, which is similar as the previous report [20]. The relative 
resistance of all samples changes consecutively with temperature as well as SWCNT film density, thickness, and post treatments. The metallic SWCNT film (sample 3) does not demonstrate metallic properties in the measured temperature range, but as all of other samples, represents the negative $\Delta \mathrm{R} / \Delta \mathrm{T}$. As we know, the resistance of SWCNT film originates from two parts: one is from individual metallic SWCNTs and the other is from the junction resistance between metallic SWCNTs. It is believed that the junction resistance between metallic SWCNTs dominates the electrical transport, which may account for the negative $(\Delta \mathrm{R} / \Delta \mathrm{T})$. Comparison of sample 1 with sample 2 reveals that the relative resistance decreases with film density. The decrease of film thickness leads to an increase in the relative resistance, as shown by the $R(T) / R(300 K)$ versus $T$ plots for samples 2 and 4 in Figure 1. Figure 1 also shows the change of relative resistance of the SWCNT films before and after thermal anneal (plots for samples 2 and $2 \mathrm{a}$, and plots for samples 4 and $4 \mathrm{a}$ ) and after further $\mathrm{HNO}_{3}$ treatment (plots for $2 \mathrm{aN}$ and $4 \mathrm{aN}$ ). It demonstrates that thermal annealed results in the increase of the relative resistance, while further $\mathrm{HNO}_{3}$ treatment will lead the decrease of the relative resistance.

Figure 2 depicts the variation in TCR for samples $1-4$ as a function of temperature, which is deduced from Figure 1 with the formula TCR $=(\Delta \mathrm{R} / \Delta \mathrm{T}) / \mathrm{R}$, where $\mathrm{R}$ is the relative resistance at temperature $\mathrm{T}$ and is shown in Figure 1. The absolute value of TCR changes with the density, thickness, and the metallic SWCNT content in SWCNT films, which originates from the different transport mechanism for these SWCNT films prepared under different parameters. By comparing the TCR versus T plots for samples 1 and 2, one can see that the magnitude of the TCR of higher density sample 2 is smaller than that of lower density sample 1 . This result reveals that the increase of SWCNT film density results in a decrease in the TCR absolute value. From the TCR versus $\mathrm{T}$ plots of samples 2 and 3, it can be seen that magnitude of TCR decreases with the increase of the metallic SWCNT content in the SWCNT film. It is postulated that the positive TCR value for individual metallic SWCNT account for the low TCR magnitude of the SWCNT film with high metallic SWCNT content [21]. The TCR versus $T$ curves for samples 2 and 4 shows the trend that the TCR absolute value for the same density of SWCNT film decreases with the thickness of the SWCNT film.

Figure 3 shows the typical TCR T plots for samples 2 and 4 before and after annealing and acid treatments. Before and after the thermal annealing, the TCR absolute values increase from 0.112 (sample 2) to 0.136 (sample 2a) and from 0.088 (sample 4) to 0.101 (sample $4 \mathrm{a}$ ) at $300 \mathrm{~K}$. The TCR absolute value for the low density SWCNT film (sample 1) at $300 \mathrm{~K}$ is 0.146 , which is the largest one among all samples, even larger than the annealed SWCNT film sample 2a, see Table 1. This result suggests that thermal annealing of sample 1 may result in even larger TCR values than that of all samples prepared here. Figure 3 shows that $\mathrm{HNO}_{3}$ treatment to the annealed samples will result in a remarkable decrease in the TCR absolute value, see the plots for samples $2 \mathrm{aN}$ and $4 \mathrm{aN}$ in Figure 3. As we know, the as-prepared SWCNT film

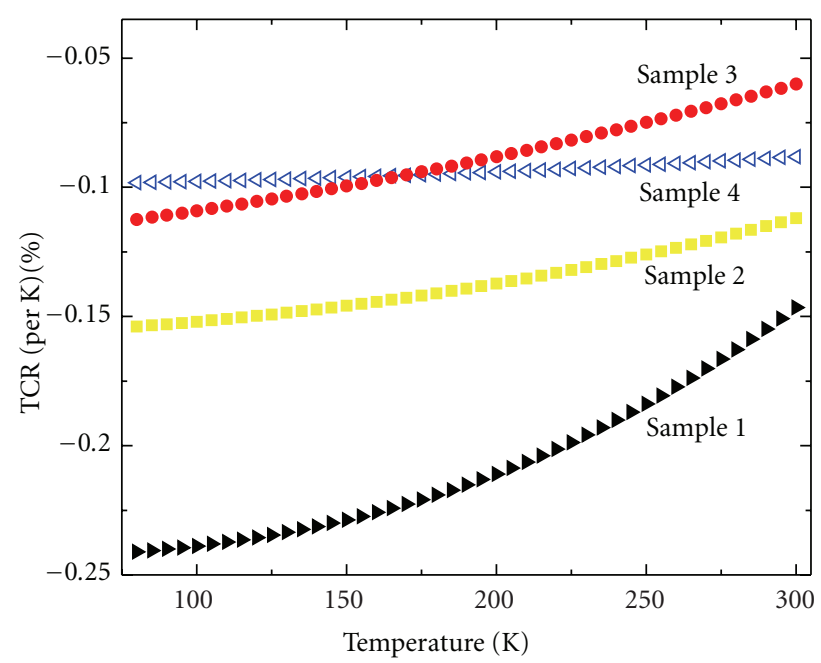

FIgURE 2: The variation of TCR for sample 1-4 as a function of temperature.

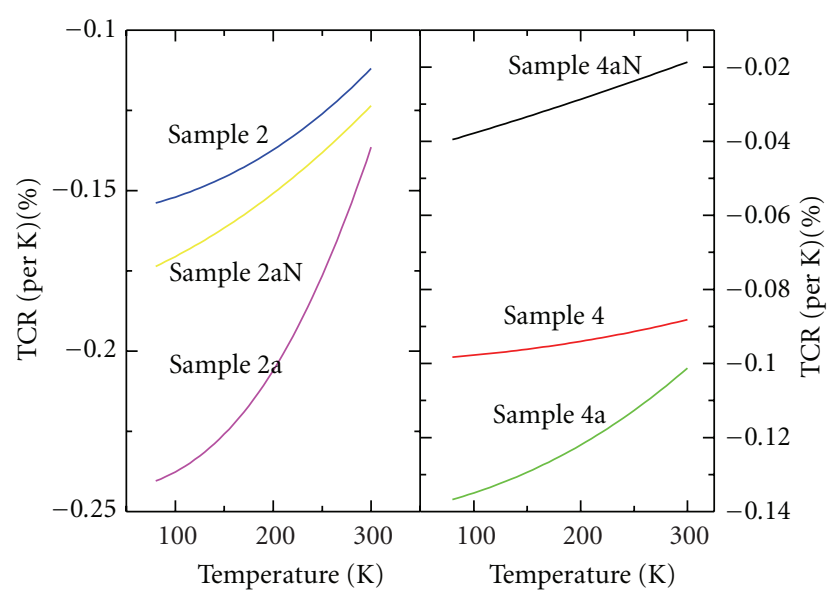

FIGURE 3: TCR $\sim$ T plots for as-prepared, annealed, and acid-treated one- and three-layered SWCNT films.

is of p-type which may originate from the acid-reflux-based purification process and atmospheric impurities, such as oxygen gas. Thermal anneal can remove the p-type dopantsand make the SWCNT more semiconducting, which may account for the increase of TCR after annealing. On the other hand, the decrease of the TCR of the acid-treated SWCNT film means that $\mathrm{HNO}_{3}$ treatment makes the annealed SWCNT film more metallic.

A carful investigation on the change of relative resistance and TCR with temperature before and after the $\mathrm{HNO}_{3}$ treatment for samples 2 and 4, as shown in Figures 1 and 3, demonstrates some differences of the electrical properties for the one- and three-layered samples. The relative resistance and TCR for sample $2 \mathrm{aN}$ are greater than those for the asprepared sample 2 but smaller than those for the annealed sample 2a. However, the relative resistance and TCR for sample $4 \mathrm{aN}$ are smaller than those of the as-prepared sample 4 and the annealed sample $4 \mathrm{a}$. The different changing trends of the relative resistance and TCR of acid-treated samples 
$2 \mathrm{aN}$ and $4 \mathrm{aN}$ may be the result of the aforementioned $2 \mathrm{D}$ to $3 \mathrm{D}$ system transition when the SWCNT film changes from one layer to three layers. The sheet resistance at room temperature for these samples as shown in Table 1 also indicates the possible $2 \mathrm{D}$ to $3 \mathrm{D}$ system transition. A more profound effect of $\mathrm{HNO}_{3}$-treatment on sample 4 than that on sample 2 is expected not only based on the transition of the SWCNT film dimensionality. Compared with sample 2, sample 4 has three layers, the SWCNTs in the same layer may be interwoven while the SWCNTs at the contact region of two adjacent layers will not interweave but have a simple contact. The intercalation of $\mathrm{HNO}_{3}$ between the layers may also account for the obvious difference of relative resistance and TCR between samples 2 and 4 upon acid treatment [22,23].

\section{Conclusions}

In this work, the low temperature electrical transport properties of the multilayered single-walled carbon nanotube (SWCNT) films have been investigated experimentally. The temperature coefficient of resistance (TCR) of SWCNT films with layered structure, controllable thickness, density and volume ratio of metallic, and semiconducting SWCNTs has been studied. The decrease of SWCNT film density, decrease of metallic SWCNT content in SWCNT film, and the thermal annealing treatment are shown to be effective approaches to obtain a SWCNT film with a large TCR absolute value. Our experimental results may provide more hints on the application of SWCNT film in bolometers.

\section{Acknowledgment}

This work was supported by the National Science Foundation under Grant DMR-0548061.

\section{References}

[1] L. Hu, D. S. Hecht, and G. Gruner, "Percolation in transparent and conducting carbon nanotube networks," Nano Letters, vol. 4, no. 12, pp. 2513-2517, 2004.

[2] Z. Yao, C. L. Kane, and C. Dekker, "High-field electrical transport in single-wall carbon nanotubes," Physical Review Letters, vol. 84, no. 13, 2000.

[3] A. Javey, H. Kim, M. Brink et al., "High- $\kappa$ dielectrics for advanced carbon-nanotube transistors and logic gates," Nature Materials, vol. 1, no. 4, pp. 241-246, 2002.

[4] Z. Wu, Z. Chen, X. Du et al., "Transparent, conductive carbon nanotube films," Science, vol. 305, no. 5688, pp. 1273-1276, 2004.

[5] C.-S. Woo, C.-H. Lim, C.-W. Cho et al., "Fabrication of flexible and transparent single-wall carbon nanotube gas sensors by vacuum filtration and poly(dimethyl siloxane) mold transfer," Microelectronic Engineering, vol. 84, no. 5-8, pp. 1610-1613, 2007.

[6] C. Lim, D.-H. Min, and S.-B. Lee, "Direct patterning of carbon nanotube network devices by selective vacuum filtration," Applied Physics Letters, vol. 91, no. 24, 2007.

[7] K. Lee, Z. Wu, Z. Chen, F. Ren, S. J. Pearton, and A. G. Rinzler, "Single wall carbon nanotubes for p-type ohmic contacts to
GaN light-emitting diodes," Nano Letters, vol. 4, no. 5, pp. 911-914, 2004.

[8] M. W. Rowell, M. A. Topinka, M. D. McGehee et al., "Organic solar cells with carbon nanotube network electrodes," Applied Physics Letters, vol. 88, no. 23, Article ID 233506, 2006.

[9] M. S. Dresselhaus, G. Dresselhaus, R. Saito, and A. Jorio, "Raman spectroscopy of carbon nanotubes," Physics Reports, vol. 409, no. 2, pp. 47-99, 2005.

[10] A. A. Green and M. C. Hersam, "Colored semitransparent conductive coatings consisting of monodisperse metallic single-walled carbon nanotubes," Nano Letters, vol. 8, no. 5, pp. 1417-1422, 2008.

[11] Z. Li, H. R. Kandel, E. Dervishi et al., "Does the wall number of carbon nanotubes matter as conductive transparent material?" Applied Physics Letters, vol. 91, no. 5, Article ID 053115, 2007.

[12] R. Lu, G. Xu, and J. Z. Wu, "Effects of thermal annealing on noise property and temperature coefficient of resistance of single-walled carbon nanotube films," Applied Physics Letters, vol. 93, no. 21, 2008.

[13] A. A. Green and M. C. Hersam, "Colored semitransparent conductive coatings consisting of monodisperse metallic single-walled carbon nanotubes," Nano Letters, vol. 8, no. 5, pp. 1417-1422, 2008.

[14] Z. Wu, Z. Chen, X. Du et al., "Transparent, conductive carbon nanotube films," Science, vol. 305, no. 5688, pp. 1273-1276, 2004.

[15] Y. L. Zhao and W. Z. Li, "Effect of annealing and $\mathrm{HNO}_{3}$-treatment on the electrical properties of transparent conducting carbon nanotube films," Microelectronic Engineering, vol. 87, no. 4, pp. 576-579, 2010.

[16] F. M. Blighe, Y. R. Hernandez, W. J. Blau, and J. N. Coleman, "Observation of percolation-like scaling-far from the percolation threshold-in high volume fraction, high conductivity polymer-nanotube composite films," Advanced Materials, vol. 19, no. 24, pp. 4443-4447, 2007.

[17] M. Penza, G. Cassano, R. Rossi et al., "Effect of growth catalysts on gas sensitivity in carbon nanotube film based chemiresistive sensors," Applied Physics Letters, vol. 90, no. 10, Article ID 103101, 2007.

[18] A. Hirsch, "Functionalization of single-walled carbon nanotubes," Angewandte Chemie, vol. 41, no. 11, pp. 1853-1859, 2002.

[19] E. B. Barros, A. G. S. Filho, V. Lemos et al., "Charge transfer effects in acid treated single-wall carbon nanotubes," Carbon, vol. 43, no. 12, pp. 2495-2500, 2005.

[20] Y. Zhou, L. Hu, and G. Grüner, "A method of printing carbon nanotube thin films," Applied Physics Letters, vol. 88, no. 12, 2006.

[21] E. Pop, D. A. Mann, K. E. Goodson, and H. Dai, "Electrical and thermal transport in metallic single-wall carbon nanotubes on insulating substrates," Journal of Applied Physics, vol. 101, no. 9, Article ID 093710, 2007.

[22] G. U. Sumanasekera, J. L. Allen, S. L. Fang, A. L. Loper, A. M. Rao, and P. C. Eklund, "Electrochemical oxidation of single wall carbon nanotube bundles in sulfuric acid," Journal of Physical Chemistry B, vol. 103, no. 21, pp. 4292-4297, 1999.

[23] Y. Wang, C. A. Di, Y. Liu et al., "Optimizing single-walled carbon nanotube films for applications in electroluminescent devices," Advanced Materials, vol. 20, no. 23, pp. 4442-4449, 2008. 

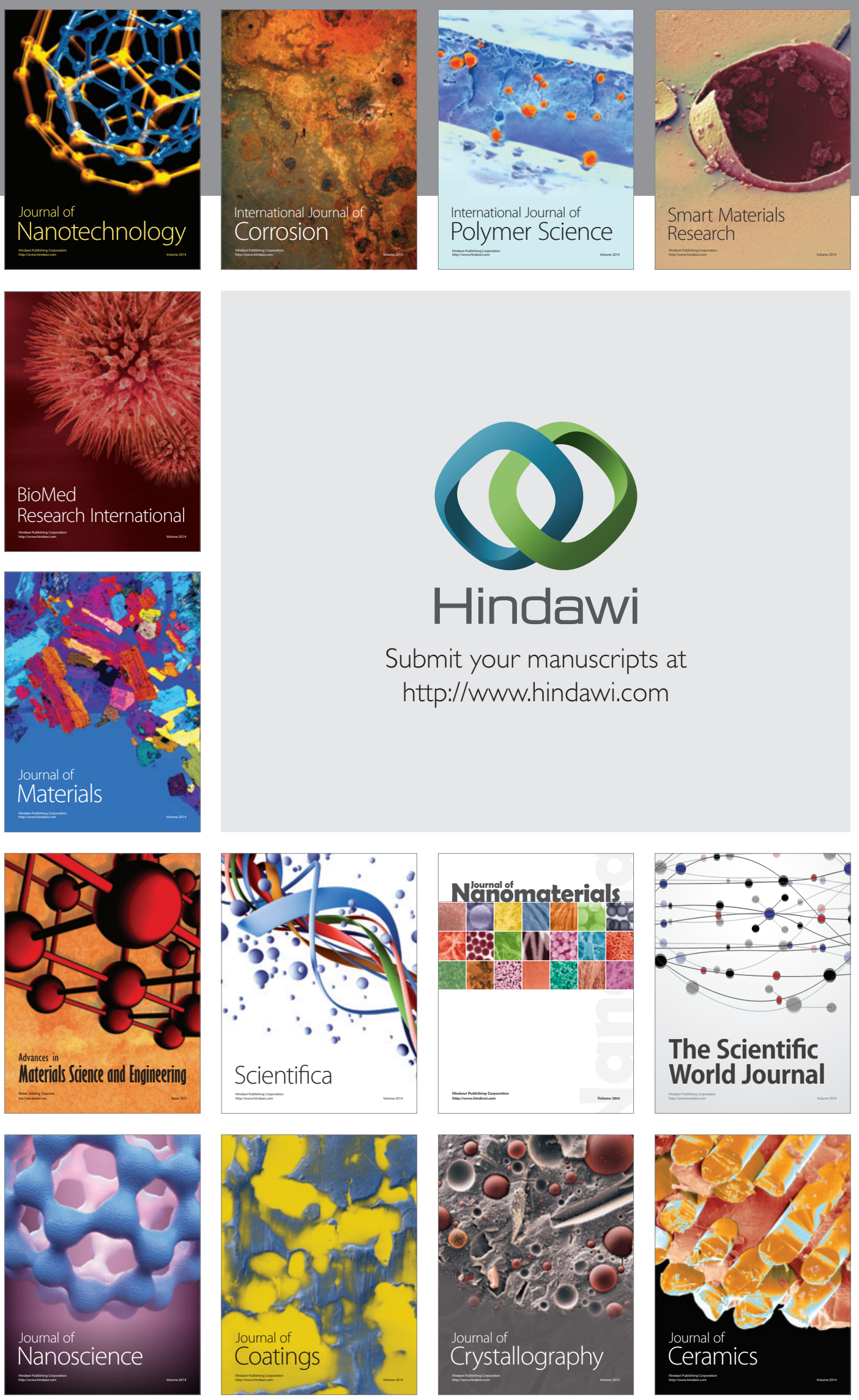

The Scientific World Journal

Submit your manuscripts at

http://www.hindawi.com

\section{World Journal}

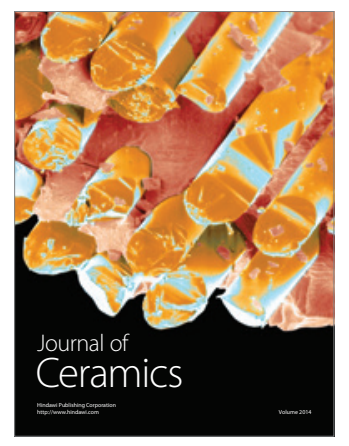

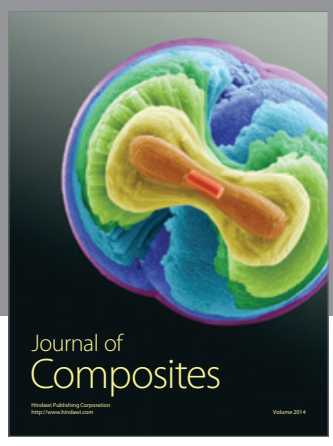
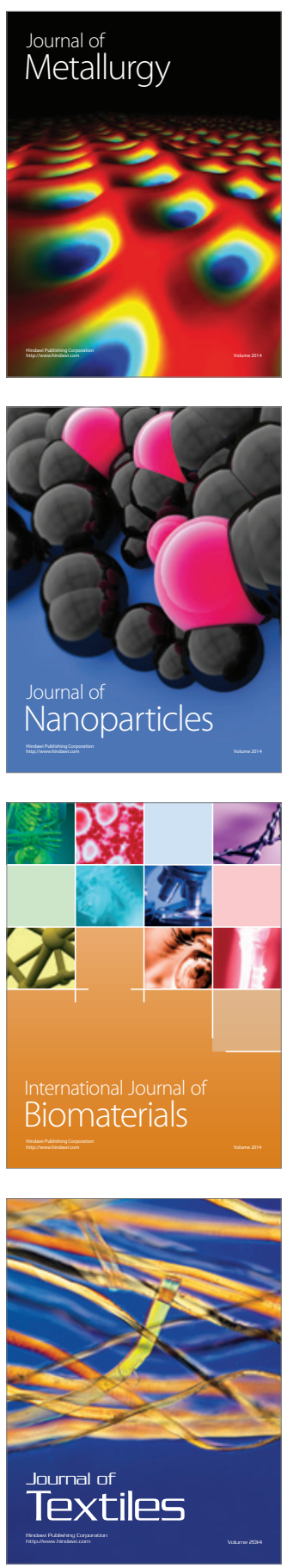